\title{
A Graph Retrieval Architecture and System for Online Sign Language Dictionary- with an application to Taiwanese Sign Language
}

\author{
Chang-Ling Hsu, Yen-Ju Tsai \\ Department of Information Management \\ Ming Chuan University \\ Taoyuan County, Taiwan \\ johnny@mail.mcu.edu.tw, hotmaneil@gmail.com
}

\author{
Ray-I Chang \\ Department of Engineering Science and Ocean Engineer \\ National Taiwan University \\ Taipei, Taiwan \\ rayichang@ntu.edu.tw
}

Received: February 5, 2021. Revised: Novemeber 4, 2021. Accepted: December 18, 2021. Published: January 3, 2022.

\begin{abstract}
Emerging applications for an online sign language dictionary require that retrieval systems retrieve a target vocabulary through visual symbols. However, when people encounter an unknown vocabulary in sign language during communication, they require the online dictionary to retrieve the vocabulary with higher recall-rate and smaller-sized graph through a mobile device. Still, three situations show that the current online dictionary needs an extension. First, previous works lack of retrieving the target graph of a vocabulary through its complete visual symbol-portfolio. Secondly, they often respond a large number of possible images; however, their precisions and recall rates remain very low. Thirdly, previous works of sign language gloves can convert the visual symbols into the graphic features, but only part of the symbols, ignoring the symbols of expression and relative direction. Therefore, the aim of this study is, based on Taiwanese Sign Language, to design a new graph retrieval architecture for sign-language (GRAS), and to implement a new graph retrieval system for sign-language (GRSS) based on this architecture. Finally, we invite users to evaluate GRSS. The experimental results show that GRSS gets convincing performance. And, GRSS adopting RDF technology can improve the performance of GRSS without adopting RDF technology.
\end{abstract}

Keywords-information retrieval; image retrieval; information system; sign language; scalable vector graphics

\section{INTRODUCTION}

Nowadays, applications of image retrieval from the World Wide Web are emerging. Search engines, such as Retrievr [16], Yahoo! Image Search [31] and Google Image [12], have been used by public users to search for similar graphs and images. Retrievr has further provided users with a rough sketching by hand to search for the target images. They respond a large number of possible images; however, their precisions and recall rates generally remain low. Still, there have been information overloading problems needed to be solved. This would result in a great loss for the fields of image retrieval, information retrieval and data mining.

Traditionally, images and graphs have been stored as a raster image format, such as bitmap, JPEG, GIF, PNG, etc., in raw compressed or original form as pixels or cells. Recently, they can be stored into an XML-based vector image format instead of a raster image format, in order to express more visual features and to reduce the size of an image. Scalable Vector Graphics (SVG) images can also optionally contain raster image and nested SVG images [29]. SVG works with three types of graphical objects: vector graphical shapes, images and text [22]. The SVG specification is an open standard developed by the World Wide Web Consortium (W3C) since 1999 [29]. SVG images and their behaviors are defined in an XML text file, with the content, features, semantic definition and interactivity, that can be indexed, searched, scripted and compressed [5, 29]. A SVG image has the following advantages after processing raster image as: (1) It has a zoom undistorted characteristics, (2) Considering the speed of transmission and processing, and storage, size of a SVG file should be minimized as small as possible, and (3) An image of lossless compression achieves good visual quality. Simple image in SVG format such as graph drawn in comics has the above advantages with smaller graph size even without containing any raster image. As we do not consider the raster images in this paper, the graphical objects are only vector graphical shapes and text. We call this kind of SVG image the SVG graph.

Sign language is used by the deaf-mute people and the others to communicate with one another. Different from other languages, words in sign language use body languages to express. Such a body language is composed of a visual symbol-portfolio, such as hand-shapes, facial expressions and movements. A word in sign language dictionary includes text symbols and visual symbols. Except for the text symbols, we can use multimedia such as graph, video, or animation to express these visual symbols.

Emerging applications for an online sign language dictionary require that retrieval systems retrieve a target vocabulary through visual symbols. However, when people encounter an unknown vocabulary in sign language during communication, they require the online dictionary to retrieve the vocabulary with higher recall-rate and smaller-sized graph 
through a mobile device. Otherwise, they are unable to retrieve the graph and specification of the vocabulary timely. Still, three situations show that the current online dictionary of sign language needs an extension.

First, Dyson and Box [8] deemed that images or graphs with an extra dimension such as their features can be used as the retrieval criteria. Elmasri and Navathe [9] have divided typical Content-based image retrieval (CBIR) techniques into three categories: (i) Category retrieval: as the images have been hierarchically classified into the previously defined categories, users must be fully aware of what the available categories. (ii) Keyword retrieval: it is about the use of keywords to retrieve images. (iii) Entity-feature-relationship retrieval: all the graphical entities in an image, their features and relationship, can be used to identify and to index the images. However, previous works about graph retrieval of sign language lack the third type. It causes users not to be able to retrieve the target SVG graph of a vocabulary of sign language through its complete visual symbol-portfolio matching the entity-feature-relationship among graphical entities. Besides, the retrieval requires an ad-hoc graphical user interface to input the symbol-portfolio of a word, and then mapping the portfolio to the features and relationship of graphical entities, and responding the matching SVG graphs and descriptions. Secondly, most of the online sign language dictionaries only retrieve the visual symbol-portfolio of sign language by text. We call it forward retrieval such as Spreadthesign.com [10] and TSL Online Dictionary [25]. Fewer online sign language dictionaries retrieve the text by symbols of sign language. We call it reverse retrieval such as ASL Dictionary [17]. The reverse retrieval uses the symbolportfolio, a combination of visual symbols such as handshapes, locations and movements. The symbol-portfolio of previous work such as ASL Dictionary responds a large number of possible images; however, its precisions and recall rates remain very low. Thirdly, previous works of sign language gloves $[13,15,27]$ can convert the visual symbols into the graphic features, but only part of the symbols of sign language, i.e. hand shape, location and movement, ignoring the other symbols, i.e. facial expressions and directions of a signer. In addition, an equipment of sign language glove is more expensive and not so popular as pervading mobile devices such as a mobile-phone or a tablet computer. A SVG graph file with smaller size and undistorted feature is very suitable for the mobile devices to retrieve graphs online.

Therefore, the aim of this study is, based on the Taiwanese Sign Language (TSL), to design a new graph retrieval architecture for sign-language (GRAS), and to implement a new graph retrieval system for sign-language (GRSS) based on this architecture. We have surveyed experts for revisions of GRAS and GRSS. Different from the previous works, GRSS uses RDF and XML Schema to represent each vocabulary of the sign-language. Finally, we invite users to use GRSS to evaluate its performance.

\section{RELATED WORK}

\section{A. Taiwanese sign language (TSL)}

Taiwanese deaf-mute population is about 133,000 people in 2011 [23]. Sign language is a visual language, different from the auditory language. Its word is defined by a limited number of basic visual symbols [25]. Due to historical factors, TSL is diverse; it is a fusion of the sign language system of Japanese and Traditional Chinese. Li [18] deems that Taiwan sign language is based on gestures in linguistics, originated from the elements of indicative symbols. In this paper, we adopt the point of $\mathrm{Li}$ that each word consists of five components including: (1) hand types, (2) orientations, (3) facial expressions, (4) movements and (5) locations. An example is shown in Figure 1. Morphology of TSL [1] is also

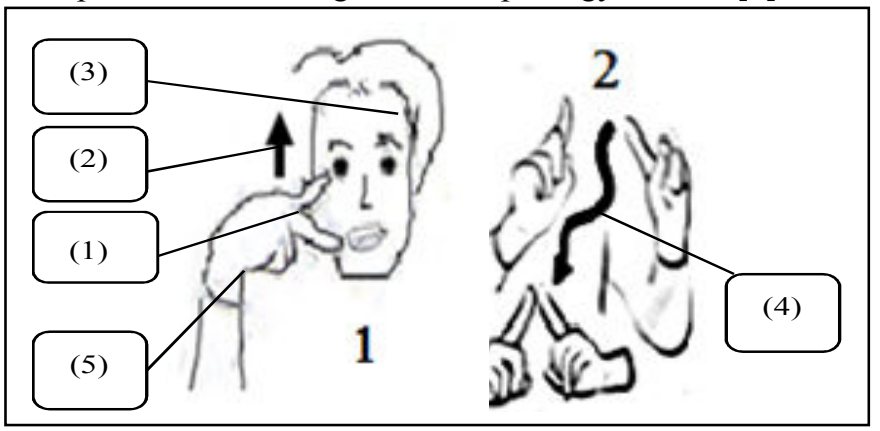

adopted by us including three word types: single word, compound word and integration words.

\section{Fig. 1. Components of a vocabulary of TSL.}

According to the characteristics of a word or the relationship between two words in TSL mentioned by Chang $[3,4]$, she categorized words into five types: (i) similar type: two words have similar gestures; (ii) combined type: two words are combined into one word; (iii) mixed-meaning type: the meanings of one and more gestures are mixed into a new meaning of one word; (iv) un-separated type: any gesture in a word cannot be removed; (v) synonymous type: the words with the same meaning but are composed of different gestures.

\section{B. $S V G$}

The "scalable" of the SVG defined by W3C means that the size of graph can zoom in or zoom out consistently [29]. SVG graphs can be scaled to adjust the original size for different screen display resolution, such as mobile phones, tablet PCs and desktop computer screen [28]. A SVG graph is composed of geometric objects such as points, lines and curves, colors, paths, coordinates and graphic structures. And raster format images, such as a GIF, PNG and JPEG are in pixels. A file size of SVG is much smaller than the one of a raster image, because the latter must be stored with each pixel.

A SVG graph is not only a graph; it is represented as the text and the graph of a sign language word. Now, let us discuss how to code both the text and the graph of a SVG graph for retrieval. Paivio [21] has proposed the dual coding theory as a theory of cognition, following the different channels of the human mind to deal with visual and text 
messages. Li, Wang and Acero [19] pointed out those previous studies about retrieving SVG image divided into: (i) Text part: matching the text by some retrieval tools. (ii) Graph part: matching among graphic objects by some retrieval tools. To sum up, we code the text and the graph separately.

\section{Retrieval Technologies}

In order to solve the graph retrieval problem, we briefly discuss the following related retrieval technologies. According to Elmasri and Navathe [9] described the image retrieval techniques in Section 1, this study adopts all the three kinds of retrieval. Besides, the technologies about how to let TSL words become exchangeable over the Internet and how to represent and link the related words need to be discussed further. As for the exchangeable part, XML Schema can be used to describe the SVG data. As XML Schema content model supports using XML for data exchange [11], a SVG file becomes exchangeable. As for the technologies about related word, RDF (Resource Description Framework) is a framework for describing web resources, such as: title, author, date and copyright information [30]. Brickley and Libby [2] proposed the tag $<$ metadata $>$ in $\mathrm{SVG}$, which supports RDF to describe the contents of the SVG. Thus, we can develop a convention of TSL for the referable resources using RDF, and we can further describe the relationships among the graphic objects in a word or among words of TSL.

The RDF expression is a triple which consists of a subject, a predicate and an object. The predicate denotes a relationship between the subject and the object. We can use the RDF expression to represent the word types of TSL. An example of combined type is that the word "eat" and "cherish" are combined into "delicious". The predicate (i.e. combined type) denotes a relationship between the subject (i.e. "delicious") and the object (i.e. "eat" and "cherish"); likewise the predicate (i.e. similar type) means the subject (i.e. "cross over") is to the object (i.e. "pass through"). As for synonymous type, there is more than one way to represent the word "discover". Therefore, GRSS can find out its related words by inference RDF expressions.

\section{RESEARCH METHODS}

The research process is shown in Fig. 2. In step 2, in order to implement GRSS, we build the architecture, GRAS, which includes a description of architecture context, information system architecture and technical architecture. In step 3, we build the initial GRSS prototype in accordance with GRAS. In step 4, experts are invited to operate the prototype system and feedback their suggestions in the review for modifying GRAS in step 5 and GRSS in step 6. In step 7, we invited users to evaluate the performance of GRSS. The evaluation has two parts: a pre-test and a post-test.

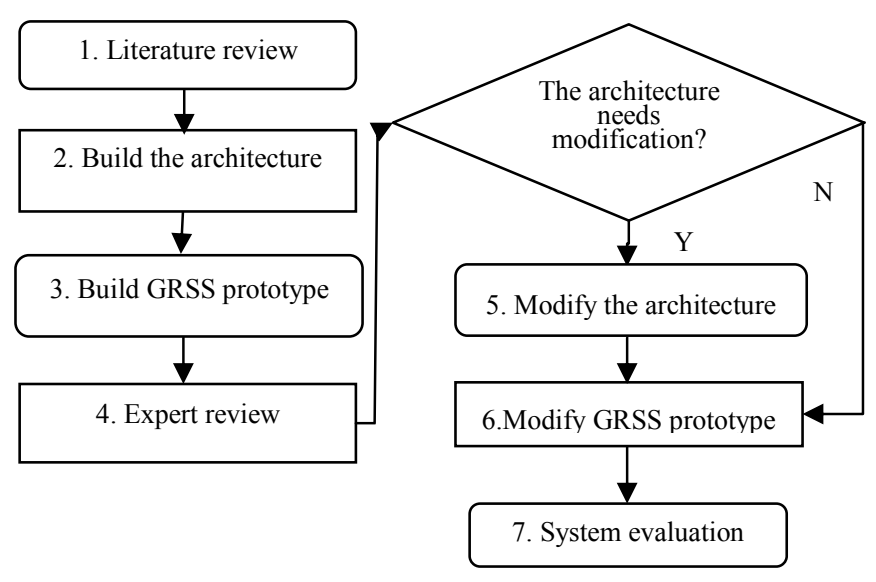

Fig. 2. Research flowchart.

\section{ARCHITECTURE DESIGN AND SYSTEM IMPLEMENTATION}

\section{A. Architecture Design}

1) Context of GRAS: The stakeholders of GRAS include dictionary editors, dictionary users and webmasters. The purpose of this architecture is to offer the stakeholders an effective use interface and system functions. This study has interviewed the stakeholders to survey their requirements that have been summed up in the following functions as: editing for sign language words, the forward retrieval and the reverse retrieval and related vocabulary. In addition, the workflows include the editing workflow, query workflow and retrieval workflow.

2) Information system architecture: The information system architecture should include two sub-architectures: application architecture and data architecture [24]. We describe both sub-architectures respectively as follows.

a) Application architecture: The system is divided into three system functions: (i) editing function: it offer editing for the editors to add, update and delete words;(ii) query function: it offer dictionary users mobile query, ordinary query, and related vocabularies;(iii) retrieval function: it supports the query function by the forward retrieval and the reverse retrieval.

b) Data architecture: As for the coding of sign language words, we design the data model of the sign language words represented in XML tree as shown in Fig. 3. We define that the relationship between a term and its related words is one to many by RDF technology. The structure of a word is composed of id, name, description, class, facial expressions, RDF and gestures. A gesture-set consists of a sequence of gestures. Each sequence contains the first gesture of the left hand and second gesture of the right hand (if it exists). And, each gesture is composed of hand types, expression, directions, movements and locations as the query criteria. Each location is represented as one of block of the block diagram of the nine-squared location, as shown in Figure 4. The diagram is divided into three main locations as: head 
range (range above the shoulders), chest range (range between the shoulder and the belly) and belly range (below the chest range). And each of the three locations is further divided into left range, middle range and right range.

3) Technology Architecture: We describe the following technologies, system software and development tools to construct GRSS, including technologies of SVG, XML, XML Schema, RDF and RDFS; the operating system: Windows 7 Professional SP1; the web server: Apache Tomcat; the browsers: Internet Explorer 8 or Mozilla Firefox; the database server: PostgreSQL; the programming languages: JSP and SQL; the tools for processing image/graph: Photoshop CS, GIMP2 and Inkscape.

\section{B. System Implementation}

We use the technology architecture to implement GRSS. An example of the screen of the retrieval for a vocabulary is displayed while execution GRSS as shown in Fig. 5.

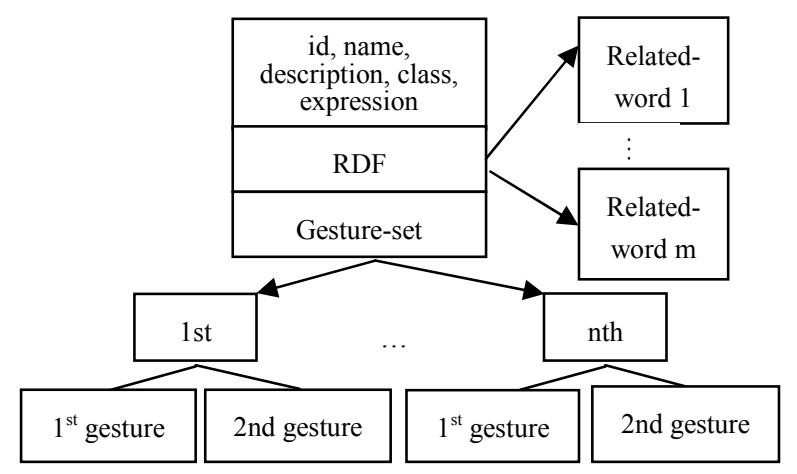

Fig. 3. XML tree of a sign language word.

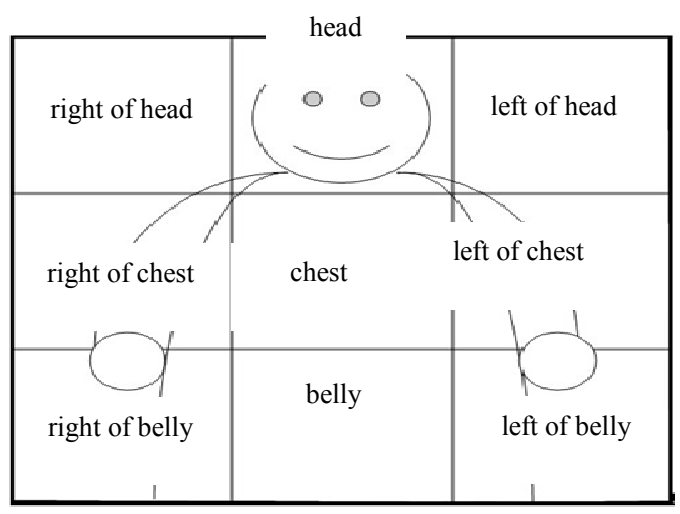

Fig. 4. Block diagram of the nine-squared location.
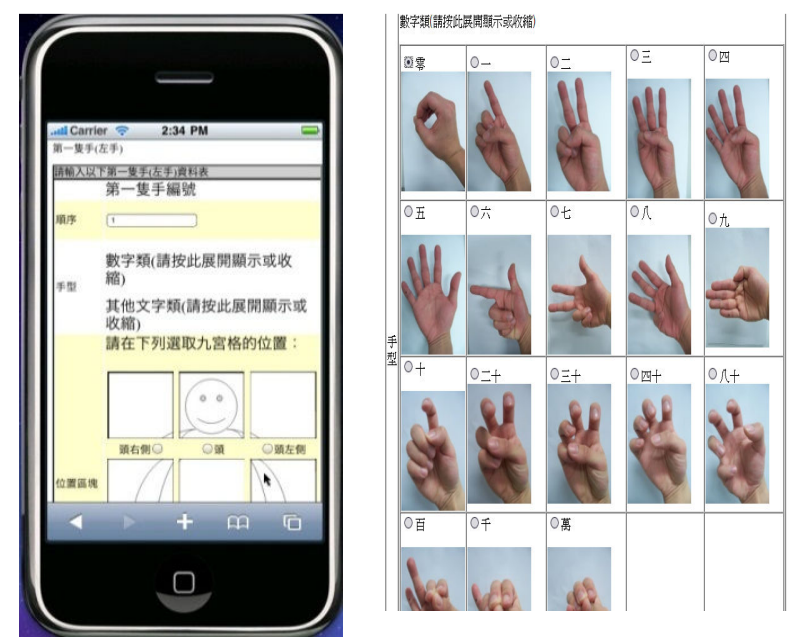

Fig. 5. The retrieval for a vocabulary of TSL using GRSS.

\section{REVIEWS AND EXPERIMENTS}

We describe the design and execution of the review for inspecting GRAS and GRSS, the establishment of the indicators for system evaluation, and the design and results of experiments respectively.

\section{A. Design and Execution of The Review}

We invite 13 experts of three different expert fields to propose amendments for GRAS and GRSS during the review. The backgrounds of the experts are shown in Table I. The documents of Technology architecture are only inspected by the IT professionals during the technical review. Except during the technical review, the documents of the application architecture and the data architecture are inspected by all the experts during the general review.

\section{B. Indicators For System Evaluation}

For evaluating the system performance, this study adopts the commonly used indicators in information retrieval, i.e. precision, recall and F-measure [7, 14, 20, 26]. Suppose there are $N$ words in the dictionary. As a user queries a word, number of all the retrieved words is $N_{R}$, and number of the retrieved words relevant to the query is $N_{w}$. In fact, number of the related words in the dictionary is $n$. The precision is calculated in Equation 1, and the recall rate depends on whether RDF relationship is taken into account. The recall rate without considering RDF relationship is calculated in Equation 2; the recall rate considering RDF relationship is calculated in Equation 3. F-measure is calculated in Equation 4.

Precision $=$

Recall rate without RDF relationship $=$

, where $n=N_{w}+R D F_{Y}+R D F_{N}, R D F_{Y}$ : number of related words predefined RDF relationship, and $R D F_{N}$ : number of related words not predefined RDF relationship.

Recall rate with RDF relationship $=\left(\mathrm{N}_{\mathrm{W}}+\mathrm{RDF}_{\mathrm{\gamma}}\right) / \mathrm{n}$. (3) 
F-measure

$(2 \times$ precision $\times$ recall) (precision + recall)

Example: Suppose we query the vocabulary "calendar" in TSL. The hand type of the left hand is "palm", and hand type of the right hand is "palm". $N_{R}$ is $8 . N_{w}$ is $1 . n$ is $3 . R D F_{Y}$ is 1 . $R D F_{N}$ is 1 . Recall rate without considering RDF relationship $=$ $1 / 3=33.33 \%$. Recall rate considering RDF relationship $=(1+$ 1) $/ 3=66.67 \%$, improves the former by $33.33 \%$.

TABLE I. BACKGROUNDS OF THE EXPERTS

\begin{tabular}{|c|c|c|c|c|c|}
\hline $\begin{array}{c}\text { Expert } \\
\text { Field } \\
\end{array}$ & Position & Seniority & $\begin{array}{c}\text { Expert } \\
\text { Field } \\
\end{array}$ & Position & Seniority \\
\hline \multirow{4}{*}{$\begin{array}{l}\text { special } \\
\text { education }\end{array}$} & teacher & 3 & \multirow{7}{*}{$\begin{array}{c}\text { IT } \\
\text { profess } \\
\text { ional }\end{array}$} & $\begin{array}{l}\text { associate } \\
\text { professor }\end{array}$ & 12 \\
\hline & teacher & 2 & & $\begin{array}{l}\text { assistant } \\
\text { professor }\end{array}$ & 4 \\
\hline & teacher & 6 & & $\begin{array}{l}\text { associate } \\
\text { professor }\end{array}$ & 25 \\
\hline & teacher & 2 & & professor & 15 \\
\hline \multirow{3}{*}{ interpreter } & interpreter & 4 & & $\begin{array}{l}\text { associate } \\
\text { professor }\end{array}$ & 15 \\
\hline & interpreter & 35 & & \multirow{2}{*}{$\begin{array}{l}\text { assistant } \\
\text { professor }\end{array}$} & \multirow[t]{2}{*}{20} \\
\hline & interpreter & 8 & & & \\
\hline
\end{tabular}

C. Design of Experiments

The Population of this study is from the TSL dictionary with graph [6] which has 2,500 words. In order to test whether the people factor influences the retrieval performances, we randomly sampled 168 words from the dictionary for 10 persons, divided into a non-deaf-mute group and a deaf-mute group in a pre-test. An attempt was made to apply GRSS to any benchmark. However, GRSS has no matching online retrieval system for TSL to compare with. Therefore, we randomly sampled 208 words from the dictionary for conducting two experiments. One experiment was for experimental group, and the other experiment was for control group. We transferred different sample data into the database of GRSS while conducting different experiment. We describe the pre-test and the experiment in detail as follows.

1) Pre-test: In order to control the influence of personal parameters, we measured the experience of sign language in the pre-test and used matching to create homogeneous groups according to the experience, deaf-mute or not, gender and education level. In order to control environmental parameters, we conducted the experiment in a browser, not in a mobile application, thus excluded an influence of tool. We divide all the 10 persons into a non-deaf-mute group with five users and a deaf-mute group with another five users for comparing the differences of inter-group and intra-group. Each user of both groups retrieved seven words per word-set, a total of 35 sample words. The experimental results show that the average precision, recall and F-measure are $63.79 \%, 41.95 \%$ and $50.62 \%$ in total for non-grouping. The average precision, recall and F-measure of both groups are $68.11 \%, 39.52 \%$ and $49.35 \%$ for deaf-mute group and $64.82 \%, 41.62 \%$ and $50.00 \%$ for non-deaf-mute group, as shown in Fig. 6. We go further in detail as: (i) the test of difference of inter-groups: As F- measure is calculated by precision and recall rate, we only compare the precision and the recall rate. At the $95 \%$ confidence level, our T-tests show that there are no significant differences in means of precision and recall between both groups. (ii) The test of difference of intra-groups: At the $95 \%$ confidence level, our F-tests of ANOVA show that there are no significant differences in means of precision and recall in each group. It shows that there is no second factor. According to these two tests, we can say that there are no significant differences among the identity of users and among individual user for the retrieval performances. In other words, it is determined that the people factor is not a confounding factor.

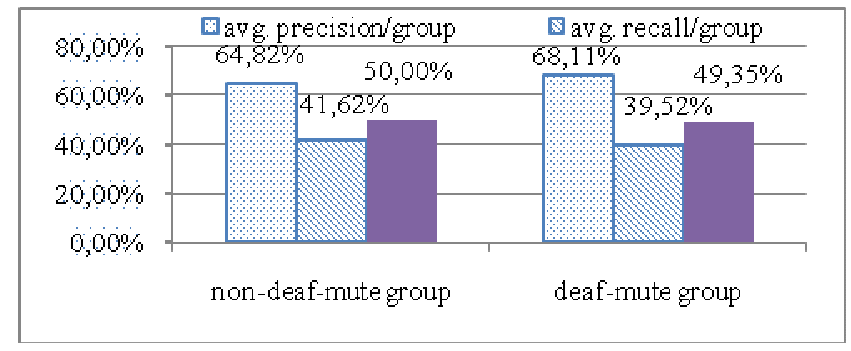

Fig. 6. Comparison of retrieval performance between both groups.

2) The experiments: As excluding an influence of the people factor, we randomly sampled 208 words from the dictionary for 23 persons in two experiments. One experiment was for retrievals of the 208 words that adopted RDF (i.e. experimental group). The other experiment was for retrievals of the 208 words that did not adopt RDF (i.e. control group). We will compare the results of both experiments in the next section.

\section{RESULTS AND DISCUSSION}

We tested 208 words by randomly sampling from the dictionary. Fig. 7 shows the experimental results for GRSS between not adopting RDF and adopting RDF. The average precision, recall and F-measure of the former are $65.98 \%$, $41.88 \%, 50.82 \%$. The average precision, recall and F-measure of latter are $65.98 \%, 83.38 \%, 72.15 \%$. The latter has improved the average recall of $41.26 \%$ than the former. The latter has improved the average F-measure of $21.33 \%$ than the former. It is clear that the experimental results show that GRSS get convincing performance. And, GRSS adopting RDF can improve the performance. 


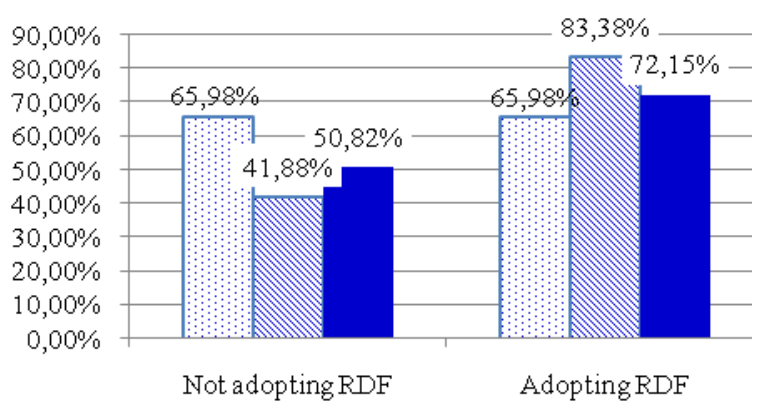

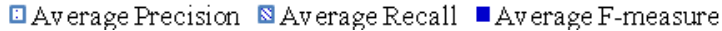

Fig. 7. Comparisons of results between not adopting RDF and adopting RDF.

\section{CONCLUSIONS}

For solving the problem of image retrieval of a vocabulary of sign language through input of symbol-portfolio of the vocabulary, we propose the graph retrieval architecture, GRAS, and the system, GRSS. GRSS responds the matching SVG graphs and descriptions well in convincing precisions, recalls and F-measures. And, GRSS adopting RDF can improve the performance of GRSS without adopting RDF.

We recommend GRAS and GRSS which will provide editors of a sign language dictionary to build an online dictionary with better function for graph retrieval. GRSS uses RDF and XML Schema to represent the SVG graph-portfolio of the vocabulary to look up the vocabulary. The proposed application for the XML Schema and the RDF, the former can be used as a reference standard for future development of local and global sign language data exchange format; while the latter for future research can further integrate Semantic Web technologies to retrieve the semantics of sign language. As for the limitation of this study, this paper's application has been limited to Taiwanese sign language; however, it will be easily expanded to any other sign languages for future studies. As GRSS can only represent and link the word relationship beforehand, about how to let GRSS find the related words automatically over the Internet is also a future work.

\section{ACKNOWLEDGMENT}

This study is financially supported by National Science Council of Taiwan under research project no. NSC-101-2221E-130-033.

\section{REFERENCES}

[1] ATSH, Fundamental Tutorial for Interpreter Guide. Taipei, Taiwan: Association of Taiwan Sound-Home (ATSH), 2007, pp. 1-2, (in Traditional-Chinese).

[2] D. Brickley and M. Libby, "Semantic webapps? lightweight RDF interfaces for SVG," in Proc. Int. Conf. on Scalable Vector Graphics (SVGOpen'04), Tokyo, Japan, Sept 2004, pp. 7-10. [Online]. Available: http://www.svgopen.org/2004/proceedings_en.html

[3] J.-H. Chang (a), "From using the Six-Books to explore the development of Taiwanese Sign Language I," Soundhome Bimonthly, pp. 11-16, 2007, (in Traditional-Chinese). [Online]. Available: http://www.soundhome.org.tw/sh_a/know/4-78-2.pdf

[4] J.-H. Chang (b), "From using the Six-Books to explore the development of Taiwanese Sign Language II," Soundhome Bimonthly, pp. 6-21, 2007, (in Traditional-Chinese). [Online]. Available: http://www.soundhome.org.tw/sh_a/know/4-79-1.pdf

[5] R.-I. Chang, Yachik Yen, Ting-Yu Hsu, "An XML-based comic image compression,” Lect. Notes Comput. Sc., vol. 5353, pp. 563-572, 2008.

[6] M. Chen, Sign Language Masters: Complete Learning Taiwanese Sign Language Manual. Taipei, Taiwan: Modern Classic Press, 2003, (in Traditional-Chinese).
[7] N. Chinchor and G. Dungca, "Four scores and seven years ago: the scoring method for MUC-6," in Proc. MUC-6 Conf., Columbia, MD, Nov. 1995, pp. 33-38 and pp. 293-316.

[8] M. C. Dyson and H. Box, "Retrieving symbols from a database by their graphic characteristics: are users consistent?," Journal of Visual Languages \& Computing, vol. 8, no. 1, pp. 85-107, 1997.

[9] R. Elmasri and S. B. Navathe, Fundamentals of Database Systems, 5th ed. Boston, MA: Addison-Wesley, 2007.

[10] ESLC. (2012) Type what you want to search. European Sign Language Center (ESLC). [Online]. Available: http://www.spreadthesign.com/

[11] B. Evjen, K. Sharkey, T. Thangarathinam, M. Kay, A. Vernet, and S. Ferguson, Professional XML, 1st ed. Hoboken, NJ: Wrox Press, 2007.

[12] Google. (2008) Google images. Google. [Online]. Available: http://www.google.com

[13] J. L. Hernandez-Rebollar, N. Kyriakopoulos, and R. W. Lindeman, "A new instrumented approach for translating American Sign Language into sound and text," in Proc. IEEE Int. Conf. Autom. Face Gesture Recognit. (FGR'04), Seoul, Korea, May 2004, pp.547 -552.

[14] G. Hripcsak and A. S. Rothschild, "Agreement, the f-measure, and reliability in information retrieval," Journal of the American Medical Informatics Association, vol. 12, no.3, pp. 296-298, 2005.

[15] T. Kuroda, Y. Tabata , A. Goto, H. Ikuta, and M. Murakami, "Consumer price data-glove for sign language recognition," in Proc. Int. Conf. Disabil., Virtual Reality \& Associated Technol. (ICDVRAT'04), Oxford, UK, Sept. 2004, pp.253-258.

[16] C. Langreiter. (2006) Retrievr. SystemOne. [Online]. Available: http://labs.systemone.at/retrievr

[17] J. Lapiak. (2000) ASL-to-English dictionary (new!). [Online]. Available: http://www.handspeak.com/word/asl-eng.php

[18] J.-H. Li, "The barrier-free environment for the deaf and the mute with sign language interpreters system," Welfare Society Quarterly of Taipei City, vol. 61, pp. 15-21, 1997, (in Traditional-Chinese).

[19] X. Li, Y.-Y. Wang, and A. Acero, "Learning query intent from regularized click graphs," in Proc. 31st ACM SIGIR Conf. on Research and Development in Information Retrieval (SIGIR'08), Singapore, July 2008.

[20] J. Makhoul, K. Francis, S. Richard, and W. Ralph, "Performance measures for information extraction," in Proc. DARPA Broadcast News Workshop, Herndon, VA, Feb. 1999, pp. 249-254.

[21] A. Paivio, "Dual coding theory: retrospect and current status," Canadian Journal of Psychology, vol. 45, no. 3, pp. 255-287, 1991.

[22] E. D. Sciascio, F. M. Donini, and M. Mongiello, "A knowledge based system for content-based retrieval of scalable vector graphics documents," in Proc. ACM symposium on Applied computing (SAC'04), Nicosia, Cyprus, Mar. 2004, pp. 1041-1044.

[23] SOMIT, "The welfare statistics of the disabled in ninth week of 2011," Weekly Bulletin of The Interior Statistics, Statistics Office of Ministry of Interior of Taiwan (SOMIT), March 2012, (in Traditional-Chinese). [Online]. http://www.moi.gov.tw/stat/news_content.aspx?sn=6009

Available:

[24] TOG, TOGAF version 9- A Pocket Guide. Reading, UK: The Open Group (TOG), 2009.

[25] J. Tsay, J. H.-Y. Tai, H.-H. Lee, Y. Chen, and Q.-Y. Yu. (2009) TSL online dictionary. Institute of Linguistics, National Chung Cheng University, Chaiyi, Taiwan. [Online]. Available: http://tsl.ccu.edu.tw/web/english/

[26] C. J. Van Rijsbergen, Information Retrieval, 2nd ed. Boston, MA: Butterworth, 1979.

[27] C. Wang, W. Gao, and S. Shan, "An approach based on phonemes to large vocabulary Chinese sign language recognition,” in Proc. IEEE Int. Conf. Autom. Face Gesture Recognit., May 2002, pp. 393-398.

[28] W3C (a). (2009) SVG Tiny 1.2. The SVG Working Group of W3C. [Online]. Available: http://www.w3.org/TR/SVGTiny12/

[29] W3C (b). (2011) What is SVG?. The SVG Working Group of W3C. [Online]. Available: http://www.w3.org/Graphics/SVG/

[30] W3schools. (2011) RDF Schema (RDFS). w3schools.com. [Online]. Available: http://www.w3schools.com/rdf/rdf_schema.asp

[31] Yahoo!. (2006) Yahoo! image search. Yahoo! Inc. [Online]. Available: http://images.search.yahoo.com

\section{Creative Commons Attribution License 4.0 (Attribution 4.0 International, CC BY 4.0)}

This article is published under the terms of the Creative Commons Attribution License 4.0 https://creativecommons.org/licenses/by/4.0/deed.en_US 